\title{
Estimación de la Edad Dental en Adultos Mediante Análisis de Relación de Área Pulpa/Diente en Premolares y Caninos Mandi- bulares Mediante Ortopantomografías, en una Muestra Chilena
}

\author{
Dental Age Estimation in Adults by Pulp/Tooth Ratio in Lower Premolars \\ and Canines by Orthopantomographs, in a Chilean Sample
}

Felipe Aguilera-Muñoz ${ }^{1,2,3}$; Sandra López-Lázaro ${ }^{1,3}$; Stefano De Luca ${ }^{4}$; Pablo Navarro-Cáceres ${ }^{1,5}$ \& Gabriel M. Fonseca ${ }^{1,3}$

AGUILERA-MUÑOZ, F.; LÓPEZ-LÁZARO, S.; DE LUCA, S.; NAVARRO-CÁCERES, P. \& FONSECA, G. M. Estimación de la edad dental en adultos mediante análisis de relación de área pulpa/diente en premolares y caninos mandibulares mediante ortopantomografías, en una muestra chilena. Int.J Morphol., 37(3):959-964, 2019.

RESUMEN: La estimación de la edad es uno de los procedimientos más importantes para la identificación humana. Cameriere et al. propusieron un método para estimación de edad evaluando la relación área pulpa/diente utilizando radiografías dentales, basado en la aposición de dentina secundaria. El objetivo de este estudio, fue desarrollar modelos de regresión lineal para la estimación de la edad, mediante la relación de área pulpa/diente en caninos y premolares mandibulares utilizando ortopantomografías (OPGs) en una población chilena de individuos adultos de edad y sexo conocidos. Se analizaron 162 OPGs digitales (67 hombres y 95 mujeres) mediante el programa ImageJ, con el fin de medir las áreas señaladas. Mediante análisis de regresión, se estudiaron las posibles interacciones entre la edad, sexo y relación de área pulpa/diente. Esta relación disminuyó regularmente con la edad y varió de 0,03 a 0,6 para premolares y 0,04 a 0,6 para caninos respectivamente. El mejor modelo de estimación de edad fue para la relación del canino inferior izquierdo obteniendo un coeficiente de determinación de R2=8,7 \%. La media de error absoluto, con un intervalo de confianza del $95 \%$, osciló entre 5,6 y 5,72 años. El análisis de ANCOVA no mostró diferencias significativas entre hombres y mujeres para la estimación de edad, al igual que para ambos lados de la cavidad oral. Según los resultados obtenidos, la relación de área pulpa/diente es una metodología fiable para la estimación de la edad en adultos. Sin embargo, los resultados demuestran que, en casos reales, es necesario contar con métodos adicionales de estimación ya que, al menos en la muestra analizada, el ajuste indicado por los coeficientes de determinación demostró falta de certeza en las estimaciones etarias producidas por los modelos de regresión lineal.

PALABRAS CLAVE: Antropología forense; Estimación de edad; Ortopantomografía; Relación área pulpa/diente.

\section{INTRODUCCIÓN}

La estimación de la edad (EE) en individuos adultos es uno de los procedimientos más importantes utilizados para establecer la identidad de individuos en diferentes contextos de investigación legal, forense o antropológica (Soomer et al., 2003). Para tales fines, se han desarrollado diferentes métodos, tanto cualitativos como cuantitativos, basados en el análisis de distintas regiones anatómicas; entre ellos, los más conocidos para la estimación de la edad en adultos son el análisis de la carilla articular iliaca (Osborne et al., 2004), de la sínfisis púbica (Suchey et al., 1988), del extremo esternal de la primera costilla y suturas craneales (Garvin \&
Passalacqua, 2012). En individuos sub adultos, destacan los métodos basados en el análisis radiográfico del carpo de la mano izquierda, desarrollo dental, y del grado de osificación de la epífisis de los huesos largos en formación (Cameriere et al., 2012b).

De los métodos de estimación de edad en adultos, es oportuno destacar aquellos basados en el análisis del desgaste dentario (Kim et al., 2000), de la transparencia de dentina radicular (Santoro et al., 2015) y de la aposición de dentina secundaria (Cameriere et al., 2007). Con respecto a

\footnotetext{
${ }^{1}$ Programa de Magíster en Odontología, Facultad de Odontología, Universidad de La Frontera, Temuco, Chile.

${ }^{2}$ Instituto de Odontoestomatología, Universidad Austral de Chile, Valdivia, Chile.

${ }^{3}$ Centro de Investigación en Odontología Legal y Forense (CIO), Facultad de Odontología, Universidad de La Frontera, Temuco, Chile.

${ }^{4}$ Área de Identificación Forense, Unidad de Derechos Humanos, Servicio Médico Legal de Chile, Santiago, Chile.

${ }^{5}$ Centro de investigación en Ciencias Odontológicas (CICO), Facultad de Odontología, Universidad de La Frontera, Temuco, Chile.
} 
este último punto, Cameriere et al. (2004), desarrollaron un método para la EE basado en el análisis de la aposición de la dentina secundaria mediante la evaluación cuantitativa de la relación de área pulpa/diente.

Como es sabido, el complejo dentino pulpar es un órgano vivo que contiene odontoblastos los que, a lo largo de la vida de un individuo, tanto por razones fisiológicas como patológicas, producen dentina secundaria la cual se va progresivamente depositando en la cámara pulpar, provocando su gradual obliteración. Este proceso mantiene una relación directa con la edad de un individuo, siendo un importante indicador biológico para la EE en adultos (Bodecker, 1925; Vasiliadis et al., 1983; Murray et al., 2002).

El método de Cameriere et al. (2004), ha sido previamente aplicado en muestras poblacionales de países como Italia (Fabbri et al., 2015), Portugal (Cameriere et al., 2013), México (De Luca et al., 2011), India (Jeevan et al., 2011), Brasil (Azevedo et al., 2015), entre otros, existiendo interés por la aplicación y validación según el contexto poblacional. Por lo mencionado anteriormente, el objetivo de este estudio fue desarrollar modelos de regresión lineal para la EE mediante la relación de área pulpa/diente (Cameriere et al., 2004) en caninos y premolares mandibulares, utilizando ortopantomografías (OPGs) en una población chilena de individuos adultos de edad y sexo conocidos.

\section{MATERIAL Y MÉTODO}

Muestras. Se analizaron 1.116 OPGs de individuos con un mínimo de 18 años de edad, residentes en la ciudad de Temuco, Chile. Las OPGs forman parte del archivo del área de imagenología de la Clínica Odontológica Docente Asistencial (C.O.D.A.) de la Facultad de Odontología de la Universidad de la Frontera. Fueron registrados el código de radiografía, sexo, fecha de nacimiento y fecha de toma radiográfica. El protocolo de investigación fue aprobado por el Comité Ético Científico de la Universidad de la Frontera, Chile (Acta No004_2017).

Se incluyeron OPGs de individuos que contasen con la presencia de los cuatro premolares y de ambos caninos inferiores, excluyendo aquellos con ausencia de al menos uno de los dientes señalados. También fueron excluidas OPGs con falta de definición y nitidez, al igual que aquellas de individuos cuyos dientes presentaran malformaciones coronarias o radiculares, tratamientos endodónticos, procesos patológicos con pérdidas de sustancia o deformaciones patológicas camerales, restauraciones, rehabilitaciones como prótesis fija unitaria u otra condición que afectase poten- cialmente los registros métricos del área pulpa/diente, de al menos uno de los premolares o de los caninos inferiores.

Mediciones en OPGs. Siguiendo el método propuesto por Cameriere et al. (2004), las imágenes radiográficas fueron archivadas en formato JPEG de alta resolución (300dpi) en un computador, las cuales fueron importadas al software gratuito de edición de imágenes ImageJ 1.5 Oi (National institutes of Health, Bethesda, MD, USA). Se ajustaron los parámetros de brillo/contraste y nitidez en caso de ser necesario, siguiendo las recomendaciones de Villanueva \& Fonseca (2011). Las imágenes fueron amplificadas para permitir la medición apropiada; la escala fue calibrada en milímetros mediante la opción "Set Scale" y, con la ayuda de la opción "Polygon Selection Tool", fueron seleccionadas las regiones del área pulpa y área diente de premolares y caninos (Fig. 1). Activando la función "Set Measurements" seleccionando "área" se obtuvieron los resultados en la sección "Results".

Acuerdo intraobservador e interobservador. Cada OPGs fue numerada de forma correlativa y ciega. Al realizar el análisis radiográfico, el observador no conocía la edad cronológica de los individuos. Todas las mediciones fueron realizadas por un mismo observador (FAM), capacitado por un antropólogo con experiencia en análisis de parámetros para la EE (SDL). Para evaluar la concordancia de las observaciones se analizó una muestra aleatoria de 20 radiografías en un intervalo de 2 semanas por un mismo observador (FAM). Para la valoración de la reproducibilidad de las observaciones, tres observadores repitieron el mismo proceso (FAM, SDL, SLL).

Luego de esto se calculó el Coeficiente de Correlación Intraclase (ICC) el que, según la clasificación propuesta por Fleiss (1981), mide el grado de acuerdo o consistencia entre medidas y se basa en un modelo de análisis de varianza con medidas repetidas (ANOVA). Se consideraron los siguientes grados de acuerdo: $<0$ sin acuerdo, $<0,4$ pobre, 0,4-0,59 moderado, 0,6-0,74 bueno, $>0,74$ excelente.

Análisis de datos. Para indicar la relación de área pulpa/ diente, se utilizó la notación de la Fédération Dentaire Internationale (FDI) del diente correspondiente. Así, por ejemplo, R35 indicó la relación entre área pulpa/diente del segundo premolar inferior izquierdo, R34 la relación de área pulpa/diente del primer premolar inferior izquierdo y así sucesivamente para R33, R45, R44 y R43. Las variables de la relación de área pulpa/diente, edad cronológica y sexo, fueron ingresadas en una plantilla de "Google Sheets". La edad cronológica, se calculó restando la fecha de nacimiento a la fecha de toma radiográfica después de haber convertido ambos a una edad decimal. 

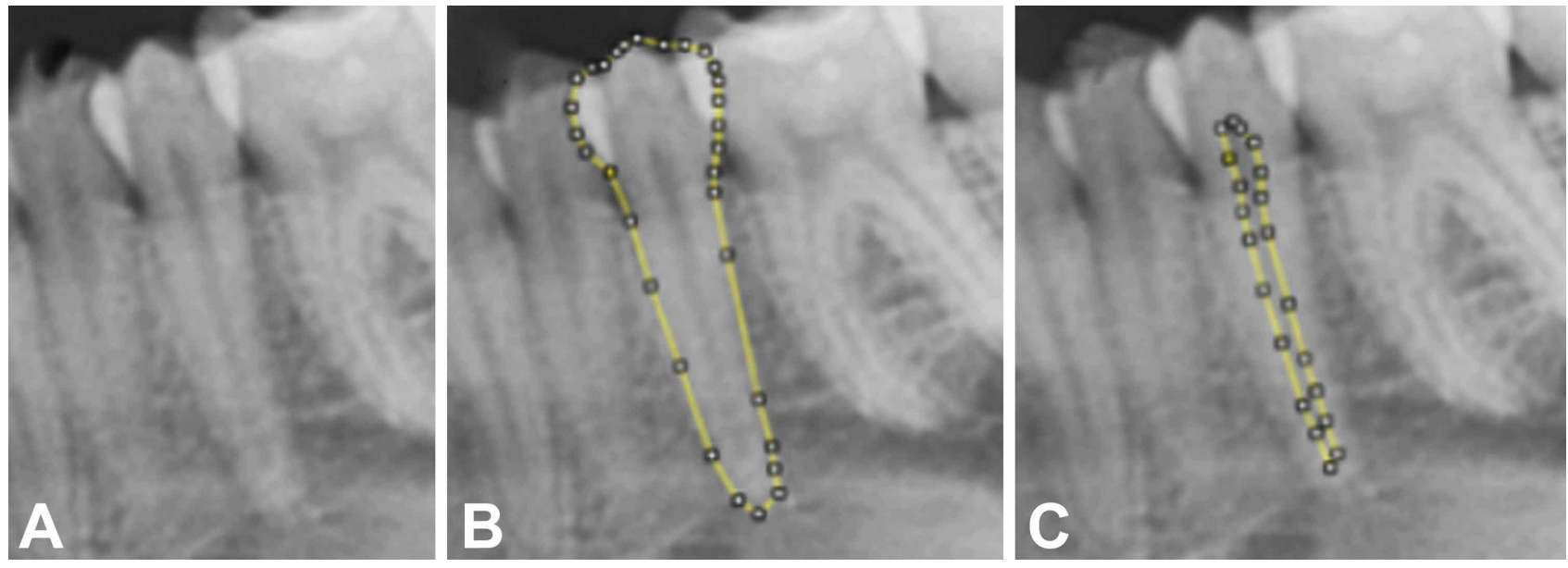

Fig. 1. Imagen radiográfica del segundo premolar inferior izquierdo (a), Medición del área del diente (b), medición del área de la pulpa (c), utilizando la herramienta lazo poligonal del software Image J.

Se desarrollaron modelos de regresión lineal simple y múltiples para estimación de edad dental. Se compararon los modelos entre sexo, grupo de caninos y premolares de ambos lados mediante el análisis de ANCOVA para establecer diferencias significativas entre los modelos, considerando la relación área pulpa/diente como covariable. Además, se comparó la edad cronológica con la edad estimada mediante el análisis de los siguientes parámetros: Coeficiente de determinación (R2), error estándar (SE) y error absoluto medio (EM).

Todos los análisis estadísticos se realizaron con el programa estadístico SPSS 15.0 (SPSS Inc., Chicago, IL), con un nivel de significancia estadística de $\mathrm{p}<0,05$.

\section{RESULTADOS}

Un total de 162 OPGs cumplió con los criterios de inclusión antes señalados y permitió realizar las mediciones para la EE dental. Tomando en consideración el sexo de los individuos muestreados, 67 OPGs correspondieron a hombres $(41,36 \%)$ y 95 a mujeres $(58,64 \%)$.

Tabla I. Distribución de edad y sexo para las muestras de OPGs estudiadas

\begin{tabular}{lllllll}
\hline \multirow{2}{*}{ Sexo } & \multicolumn{7}{c}{ Edad de corte } \\
\cline { 2 - 7 } & $18-28$ & $29-38$ & $39-48$ & $49-58$ & $59-68$ & $>69$ \\
\hline Hombre & 59 & 4 & 1 & 0 & 1 & 2 \\
Mujer & 80 & 7 & 7 & 0 & 1 & 0 \\
Total & 139 & 11 & 8 & 0 & 2 & 2 \\
\hline
\end{tabular}

No hubo diferencias estadísticamente significativas al realizar las mediciones intra e interobservador y el ICC fue $>0,76$, lo cual reveló un grado de confiabilidad excelente, según lo establecido en la clasificación de Fleiss.

El rango de edad varió entre los 18 y 79 años. La frecuencia de personas con dientes ausentes aumentó con la edad, siendo el grupo entre los 18 y 28 años, donde más se concentraron las muestras. La distribución según sexo y edad se observa en la Tabla I.

La relación de área pulpa/diente para premolares disminuyó regularmente con la edad y varió de 0,03 a 0,6 , al igual que para los caninos la cual varió de 0,04 a 0,6. La Figura 2, muestra las relaciones de área pulpa/ diente de caninos y premolares según edades.

En el análisis de regresión desarrollado, se obtuvieron valores de $\mathrm{R} 2$ de 7,9\%,0 \% y 8,7\% respectivamente, para el segundo premolar inferior izquierdo (R35), primer premolar inferior izquierdo (R34) y el canino inferior izquierdo (R33). En el caso del segundo premolar inferior derecho (R45), el primer premolar inferior derecho (R44) y el canino inferior derecho (R33), los resultados de R2 fueron $0,0 \%, 0,7 \%$ y $0,5 \%$ respectivamente. Estos resultados demostraron que el mejor modelo de estimación de edad fue para R33 (R2=8,7 \%). En los análisis de regresiones múltiples para las relaciones de R35+R34+R33, R45+R44+R43 y R35+R34+R33+R45+R44+R43, el R2 fue de $0,4 \%, 0,4 \%$ y $0,6 \%$ respectivamente. Estos análisis de regresión produjeron varias fórmulas, las cuales se subdividieron según el número y ubicación de caninos y premolares inferiores. Solo los modelos de regresión R35, R33 y R44 mostraron diferencias estadísticamente significativas. La 


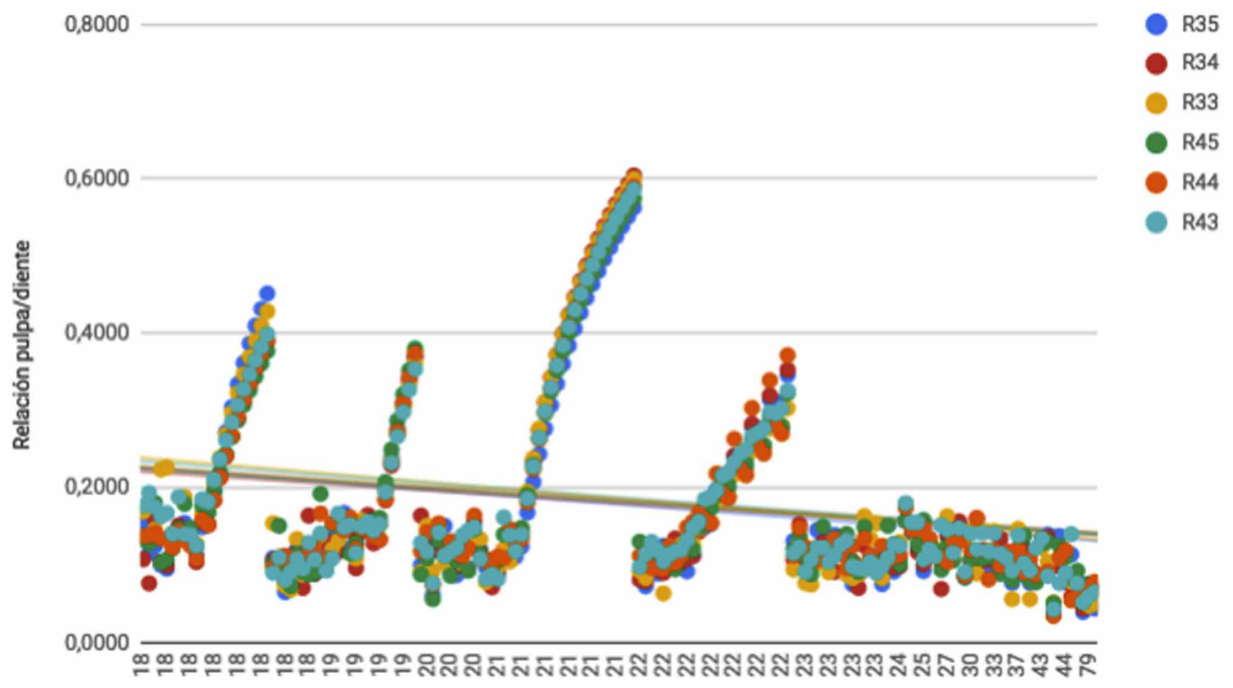

Fig. 2. Diagrama de dispersión que muestra las relaciones de área pulpa/diente $(\mathrm{R})$ de caninos y premolares inferiores, por edad.

Tabla II. Ecuación de regresión utilizando premolares y caninos inferiores.

\begin{tabular}{llllll}
\hline Relación & Ecuación & $\mathrm{R}^{2}$ & SE & EM & $\mathrm{p}$ \\
\hline R35 & edad $=28,184-23,381 \mathrm{x}$ & 0,079 & 9,35 & 5,66 & 0,00 \\
R34 & edad $=23,916+0,313 \mathrm{x}$ & 0,006 & 9,77 & 5,60 & 0,75 \\
R33 & edad $=28,271-22,887 \mathrm{x}$ & 0,087 & 9,34 & 5,72 & 0,00 \\
R45 & edad $=23,904+0,327 \mathrm{x}$ & 0,005 & 9,77 & 5,59 & 0,66 \\
R44 & edad $=28,286-23,248 \mathrm{x}$ & 0,077 & 9,36 & 5,70 & 0,00 \\
R43 & edad $=23,907+0,322 \mathrm{x}$ & 0,005 & 9,77 & 5,60 & 0,69 \\
\hline
\end{tabular}

media de error absoluto, con un intervalo de confianza del $95 \%$, osciló entre 5,6 y 5,72 años (Tablas II y III).

No existieron diferencias significativas en la relación de área pulpa/diente de caninos y premolares entre hombres y mujeres ( $\mathrm{p}>0,05)$, lo que indicó que el sexo no tuvo un efecto significativo en las variables, al igual que las relaciones entre el lado izquierdo (R35+R34+R33) y derecho (R45+R44+R33) de la cavidad oral.

\section{DISCUSIÓN}

La estimación de la edad es uno de los pilares básicos del proceso de identificación humana (Komar \& Buikstra, 2008). Debido a los desafíos inherentes al envejecimiento de un individuo, y la necesidad de una mayor fiabilidad en las metodologías de estimación de la edad en individuos adultos, los métodos deben ser convenientemente validados en distintas poblaciones.

En Chile, se ha aplicado el método de Cameriere et al. (2008) solamente para estimar la mayoría de edad (18 años), en base al análisis de la relación entre el ápice abierto de la raíz y la longitud del tercer molar; los resultados obtenidos confirmaron la fiabilidad y reproducibilidad del método (Cameriere et al., 2018), corroborando la validez de éste para la población Chilena. Sin embargo, al menos en la literatura consultada por los autores, en Chile no se encuentran antecedentes bibliográficos de la aplicación del método de aposición de dentina secundaria para la estimación de la edad en adultos.

Tabla III. Ecuación de regresión utilizando más de dos dientes inferiores.

\begin{tabular}{llllll}
\hline Relación & Ecuación & $\mathrm{R}^{2}$ & $\mathrm{SE}$ & $\mathrm{EM}$ & $\mathrm{p}$ \\
\hline R35+R34+R33 & edad=24.455-0.72x & 0.004 & 9.75 & 5,67 & 0.431 \\
R45+R44+R43 & edad=23.994+0.013x & 0.004 & 9.77 & 5,64 & 0.986 \\
R35+R34+R33+R45+R44+R43 & edad=24.038-0.041x & 0.006 & 9.77 & 5,64 & 0.910
\end{tabular}

R2, coeficiente de determinación; SE, error estándar; EM, error absoluto medio; p, significancia estadística. 
AGUILERA-MUÑOZ, F.; LÓPEZ-LÁZARO, S.; DE LUCA, S.; NAVARRO-CÁCERES, P. \& FONSECA, G. M. Estimación de la edad dental en adultos mediante análisis de relación de área pulpa/diente en premolares y caninos mandibulares mediante ortopantomografías, en una muestra chilena. Int. J Morphol., 37(3):959-964, 2019.

En este estudio, se incluyeron exclusivamente radiografías de caninos y premolares inferiores, lo que puede originar inconvenientes si se pretende analizar grupos de edad más avanzada por la dificultad de conseguir radiografías que cumplan con los criterios de inclusión, ya que las tasas de edentulismo en la población Chilena son cada vez más altas a medida que aumenta la edad cronológica del individuo (Ministerio de Salud, 2010).

La relación de área pulpa/diente para premolares y caninos disminuyó regularmente con la edad, ya que los valores más bajos se produjeron en sujetos mayores. Esto concuerda con estudios anteriores que se reportaron resultados similares al evaluar premolares inferiores mediante OPGs (Cameriere et al., 2012a), o radiografías periapicales de caninos (Jeevan et al.). Anastácio et al. (2018) y Paewinsky et al. (2005) también indicaron una relación inversa entre la edad y la relación área pulpa/diente, encontrando valores más bajos en sujetos mayores.

Con respecto al coeficiente de determinación, en este estudio se obtuvieron valores considerablemente bajos, con cifras más altas en los modelos de regresión simple que en los de regresión múltiple. Esto indicaría una baja fiabilidad de los modelos obtenidos, siendo el valor de R2 mayor en el análisis de regresión para el canino inferior izquierdo. Si bien estudios similares han reportado altos coeficientes de determinación para este método (Cameriere et al., 2004; De Luca et al.; Cameriere el at., 2012a; Fabbri et al.), otros reportes, como el de Anastácio et al., mostraron valores bajos en los resultados obtenidos. Cabe mencionar que, con frecuencia, el coeficiente R2 puede dar una idea equivocada de las capacidades predictivas del modelo de estimación propuesto, ya que representa exclusivamente un coeficiente con capacidades explicativas de este (Martínez Rodríguez, 2005). Por esta razón, para su interpretación, se debe evaluar siempre el grado de significación (p), el tamaño de la muestra y el número de variables explicativas incluidas en el modelo de regresión (Martínez Rodríguez). En el caso de este estudio, la mayor presencia de individuos jóvenes con respecto a aquellos de edad más avanzada, podría estar directamente relacionada con la mayor falta de certeza de las estimaciones etarias, producidas por los modelos de regresión lineal obtenidos. Por lo expresado anteriormente, el análisis de la magnitud media del error relativo, o absoluto, puede ser una herramienta estadística más conveniente para la evaluación de un modelo de regresión. De hecho, en este estudio, los promedios del valor de error medio absoluto oscilaron entre 5,6 y 5,72, demostrando una capacidad predictiva aceptable.

Siguiendo a Cameriere et al. (2004, 2012a) y Paewinsky et al., los resultados obtenidos para el coeficiente de determinación podrían deberse a varios factores: el ta- maño de la muestra, la técnica de medición utilizada, el software usado para las mediciones, y finalmente la variabilidad geográfica de la población analizada. Por otro lado, los resultados obtenidos en este estudio mostraron un alto grado de acuerdo intra- e interobservador, lo que indica una alta reproducibilidad del método y, por ende, la replicabilidad de las mediciones por parte de otros observadores diferentes al que desarrollaron la metodología analizada.

Al igual que en reportes previos (Cameriere et al., 2012a; Azevedo et al.; Dehghani et al., 2018), en este estudio se demostró que la variable sexo no tuvo un efecto significativo en el modelo utilizado. Además, los resultados obtenidos mediante las regresiones múltiples demostraron que se pueden incluir dientes independientemente del sector de la cavidad oral, para aumentar la muestra e intentar incrementar la fiabilidad y precisión del método.

Dentro de las limitaciones de este estudio, puede mencionarse la distorsión y la falta de nitidez producidas de forma inherente en una ortopantomografía (Zúñiga, 2017), lo que podría generar errores en las mediciones de las áreas de la pulpa y del diente. En ocasiones, el operador puede tener dificultades en sus mediciones debido a la calidad de las imágenes en los límites más apicales del diente y en el área de la pulpa. Una mejora de estos parámetros, o el uso de radiografías periapicales (Cameriere et al., 2007, 2009; Zaher et al., 2011; Cameriere et al., 2013) podría reducir el error de estimación y mejoraría la estimación de edad dental.

CONCLUSIONES. De acuerdo a estos resultados, se puede afirmar que la relación de área pulpa/diente para medir la aposición de la dentina secundaria en canino y premolares, es una metodología fiable para estimar la edad en adultos según lo propuesto por Cameriere et al. (2004). Sin embargo, los resultados de este estudio demuestran que, en casos reales, se hace necesario contar con métodos adicionales de estimación ya que, al menos en la muestra analizada, el ajuste indicado por los coeficientes de determinación mostró incerteza en las estimaciones etarias producidas por los modelos de regresión lineal.

AGUILERA-MUÑOZ,F.; LÓPEZ-LÁZARO, S.; DE LUCA, S.; NAVARRO-CÁCERES, P. \& FONSECA, G. M. Dental age estimation in adults by pulp/tooth ratio in lower premolars and canines by orthopantomographs, in a Chilean sample. Int. J. Morphol., 37(3):959-964, 2019.

SUMMARY: Age estimation is one of the most important procedures for human identification. Cameriere et al. proposed a method for age estimation based on the analysis of the pulp/tooth ratio by using dental $\mathrm{x}$-rays, based on the apposition of secondary dentine. The objective of this study was to develop linear regression models for age estimation, by means of the pulp/tooth ratio of 
mandibular canines and premolars using orthopantomographies (OPGs) in a Chilean population of adults of known age and sex. We analyzed 162 digital OPGs (67 men and 95 women) through ImageJ software, in order to measure these areas. By means of regression analysis, the possible interactions between age, sex and pulp / tooth ratio were studied. This relationship decreased regularly with age and ranged from 0.03 to 0.6 for premolars and 0.04 to 0.6 for canines, respectively. The best model of age estimation was for the relation of the lower left canine, obtaining a coefficient of determination of R2=8.7 $\%$. The mean absolute error, with a confidence interval of $95 \%$, ranged between 5.6 and 5.72 years. The ANCOVA analysis showed no significant differences between men and women for age estimation, as well as for both sides of the oral cavity. According to the results, the pulp/tooth ratio is a reliable methodology for estimating age in adults. However, the results showed that, in actual cases, it is necessary to use additional estimation methods since, at least in the sample analyzed, the adjustment indicated by coefficients of determination showed uncertainty in age estimation produced by the linear regression models.

KEY WORDS: Forensic anthropology; Age estimation; Ortopanthomography; Pulp/tooth ratio.

\section{REFERENCIAS BIBLIOGRÁFICAS}

Anastácio, A. C.; Serras, C.; Vargas de Sousa Santos, R. F. \& Palmela Pereira, C. Validation of Cameriere's medical-legal age estimation method using seconds premolars in a Portuguese population. J. Forensic Leg. Med., 60:304, 2018 .

Azevedo, A. de C.; Alves, N. Z.; Michel-Crosato, E.; Rocha, M.; Cameriere, R. \& Biazevic, M. G. Dental age estimation in a Brazilian adult population using Cameriere's method. Braz. Oral Res., 29(1):1-9, 2015.

Bodecker, C. F. A consideration of some of the changes in the teeth from young to old age. Dent. Cosm., 67:543-9, 1925.

Cameriere, R.; Cunha, E.; Sassaroli, E.; Nuzzolese, E \& Ferrante, L. Age estimation by pulp/tooth area ratio in canines: study of a Portuguese sample to test Cameriere's method. Forensic Sci. Int., 193(1-3):128.e1-6, 2009.

Cameriere, R.; Cunha, E.; Wasterlain, S. N.; De Luca, S.; Sassaroli, E.; Pagliara, F.; Nuzzolese, E.; Cingolani, M. \& Ferrante, L. Age estimation by pulp/ tooth ratio in lateral and central incisors by peri-apical X-ray. J. Forensic Leg. Med., 20(5):530-6, 2013.

Cameriere, R.; De Luca, S.; Alemán, I.; Ferrante, L. \& Cingolani, M. Age estimation by pulp/tooth ratio in lower premolars by orthopantomography. Forensic Sci. Int., 214(1-3):105-12, 2012a.

Cameriere, R.; De Luca, S.; Biagi, R.; Cingolani, M.; Farronato, G. \& Ferrante, L. Accuracy of three age estimation methods in children by measurements of developing teeth and carpals and epiphyses of the ulna and radius. $J$. Forensic Sci., 57(5):1263-70, 2012b.

Cameriere, R.; Ferrante, L. \& Cingolani, M. Variations in pulp/tooth area ratio as an indicator of age: a preliminary study. J. Forensic Sci., 49(2):317-9, 2004.

Cameriere, R.; Ferrante, L.; Belcastro, M.G.; Bonfiglioli, B.; Rastelli, E. \& Cingolani, M. Age estimation by pulp/tooth ratio in canines by mesial and vestibular peri-apical X-rays. J. Forensic Sci., 52(5):1151-5, 2007.

Cameriere, R.; Ferrante, L.; De Angelis, D.; Scarpino, F. \& Galli, F. The comparison between measurement of open apices of third molars and Demirjian stages to test chronological age of over 18 year olds in living subjects. Int. J. Legal Med., 122(6):493-7, 2008.

Cameriere, R.; Velandia Palacio, L.A.; Pinares, J.; Bestetti, F.; Paba, R.; Coccia, E. \& Ferrante, L. Assessment of second (I2M) and third (I3M) molar indices for establishing 14 and 16 legal ages and validation of the Cameriere's I3M cut-off for 18 years old in Chilean population. Forensic Sci.Int., 285:205.e1205.e5, 2018.

De Luca, S.; Bautista, J.; Alemán, I. \& Cameriere, R. Age-at-death estimation by pulp/tooth area ratio in canines: study of a 20th-century Mexican sample of prisoners to test Cameriere's method. J. Forensic Sci., 56(5):1302-9, 2011.

Dehghani, M.; Shadkam, E.; Ahrari, F. \& Dehghani, M. Age estimation by canines' pulp/tooth ratio in an Iranian population using digital panoramic radiography. Forensic Sci. Int., 285:44-9, 2018.

Fabbri, P. F.; Viva, S.; Ferrante, L.; Lonoce, N.; Tiberi, I. \& Cameriere, R. Radiological tooth/pulp ratio in canines and individual age estimation in a sample of adult neolithic skeletons from Italy. Am. J. Phys. Anthropol. 158(3):423-30, 2015

Fleiss, J. L. Statistical Methods for Rates and Proportions. New York, John Wiley, 1981.

Garvin, H. M. \& Passalacqua, N. V. Current practices by forensic anthropologists in adult skeletal age estimation. J. Forensic Sci., 57(2):427-33, 2012.

Jeevan, M. B.; Kale, A. D.; Angadi, P. V. \& Hallikerimath, S. Age estimation by pulp/tooth area ratio in canines: Cameriere's method assessed in an Indian sample using radiovisiography. Forensic Sci Int., 204(1-3):209.e1-5, 2011.

Kim, Y. K.; Kho, H. S. \& Lee, K. H. Age estimation by occlusal tooth wear. J. Forensic Sci., 45(2):303-9, 2000.

Komar, D. A. \& Buikstra, J. E. Forensic Anthropology: Contemporary Theory and Practice. New York, Oxford University Press, 2008.

Martínez Rodríguez, E. Errores frecuentes en la interpretación del coeficiente de determinación lineal. An. Jurid. Econ. Escur., 38:315-32, 2005.

Ministerio de Salud (MINSAL). Análisis de Situación de Salud Bucal en Chile. Santiago de Chile, Ministerio de Salud, Gobierno de Chile, 2010.

Murray, P.E.; Stanley, H.R.; Matthews, J.B.; Sloan, A.J. \& Smith, A.J. Agerelated odontometric changes of human teeth. Oral Surg. Oral Med. Oral Pathol. Oral Radiol. Endod., 93(4):474-82, 2002.

Osborne, D. L.; Simmons, T. L. \& Nawrocki, S. P. Reconsidering the auricular surface as an indicator of age at death. J. Forensic Sci., 49(5):905-11, 2004.

Paewinsky, E.; Pfeiffer, H.\& Brinkmann, B. Quantification of secondary dentine formation from orthopantomograms--a contribution to forensic age estimation methods in adults. Int. J. Legal Med., 119(1):27-30, 2005.

Santoro, V.; Fiandaca, C.; Roca, R.; Marini, C.; De Donno, A. \& Introna, F. Validity Comparison of Three Dental Methods for Age Estimation Based on Tooth Root Translucency. J. Forensic Sci., 60(5):1310-5, 2015.

Soomer, H.; Ranta, H.; Lincoln, M. J.; Penttilä, A. \& Leibur, E. Reliability and validity of eight dental age estimation methods for adults. J. Forensic Sci., 48(1):149-52, 2003

Suchey, J. M.; Brooks, S. T. \& Katz, D. Instructions for use of the Suchey Brooks system for age determination of the female os pubis. Instructional materials accompanying female pubic symphyseal models of the Suchey-Brooks system. Colorado, France Casting, 1988.

Vasiliadis, L.; Darling, A. I. \& Levers, B. G. The amount and distribution of sclerotic human root dentine. Arch. Oral Biol., 28(7):645-9, 1983.

Villanueva, J. C. \& Fonseca, G. M. Digitalización de radiografías panorámicas en la rutina de odontólogos de la Provincia de Córdoba, Argentina. Aseguramiento de calidad para procedimientos de identificación forense. F. O.P. J., 2(5):10-4, 2011

Zaher, J. F.; Fawzy, I. A.; Habib, S. R. \& Ali, M. M. Age estimation from pulp/ tooth area ratio in maxillary incisors among Egyptians using dental radiographic images. J. Forensic Leg. Med., 18(2):62-5, 2011.

Zúñiga, J. Quantification by quadrants of the distortion present in conventional panoramic radiograph. Int. J. Morphol., 35(1):265-72, 2017.

\section{Dirección para correspondencia: \\ Dr. Gabriel M. Fonseca}

Centro de Investigación en Odontología Legal y Forense (CIO)

Facultad de Odontología

Universidad de La Frontera

Francisco Salazar 01145

Temuco - CHILE

\section{Email: gabriel.fonseca@ufrontera.cl}

Recibida : 28-01-2019

Aceptado: 14-03-2019 